\title{
A Retrospective Comparative Study to Evaluate the Clinical Efficacy Using a Bioactive Glass Ceramic Spacer in Posterior Lumbar Interbody Fusion
}

\author{
Chan Hee Shin, Sung Hwan Hwang, Jang Hun Kim, Sang Hoon Yoon, Byung-Kyu Cho \\ Department of Neurosurgery, Armed Forces Capital Hospital, Seongnam, Korea
}

Received: May 30, 2021

Accepted: July 6, 2021

Corresponding Author:

Sang Hoon Yoon, M.D.

Department of Neurosurgery,

Armed Forces Capital Hospital

81, Saemaeul-ro 177th-gil,

Bundang-gu, Seongnam 13574,

Korea

Tel: +82-31-725-6236

Fax: +82-31-706-0987

E-mail:arch73@gmail.com

\begin{abstract}
Objective
Considering the advancement of bone graft substitution, the authors aimed to achieve clinical safety by simultaneously using one bioactive ceramic spacer (NovoMax) and a conventional polyetheretherketone (PEEK) cage at first use. We compared the results between different cages, even in the same disc space and to the control group using only conventional PEEK cages.

Methods

This retrospective analysis included data from patients who underwent posterior lumbar interbody fusion surgery between 2015 and 2017. The hybrid group ( $\mathrm{H}$ group) included 19 patients with 21 lumbar disc levels, and the control group was defined as the PEEK group (P group), which included 16 patients with 18 disc levels. The degree of fusion was confirmed on computed tomography based on the fusion grade system and dynamic X-rays. We analyzed the fusion rate between the two groups using Cox proportional hazard model.

Results

There were 14 cases (72.2\%) in Group P and 15 cases (71.4\%) in Group H with confirmed complete union during the observation period $(\mathrm{p}=0.62)$. The relative hazard of incomplete fusion with osteolysis in both the $\mathrm{P}$ and $\mathrm{H}$ groups was increased by 5.41 times ( $\mathrm{p}=0.004$, confidence interval 1.704-17.204). In the $H$ group, significant instrument-related complications, such as fragmentation, slippage, subsidence, and osteolysis were observed in the Novomax cage.

Conclusions

Using NovoMax could result in osteolysis, cage migration, fragmentation, or subsidence. The use of NovoMax is expected to be compatible for bone fusion surgery, but close attention should be paid to some problems that may accompany it.
\end{abstract}

Keywords: Posterior lumbar fusion; Bioactive glass ceramics spacer; Polyetheretherketone cage; Fusion rate 


\section{INTRODUCTION}

Posterior lumbar interbody fusion (PLIF) is commonly used to prevent posterior column instability or nerve compression, related to spondylolisthesis, spinal stenosis, or traumatic injury. Compared with the posterior or posterolateral fusion method, PLIF is a biomechanical method that disperses the working load to the anterior column of the vertebral body ${ }^{1-3)}$. The inserted cage plays an important role as a spacer to expand disc height and prevent intervertebral foraminal stenosis ${ }^{4}$. Weight can be dispersed to the anteriorly positioned interbody cage, and it is the most potent method for augmenting intervertebral disc space.

A degenerative disc can cause severe back pain, and interbody fusion can eliminate axial pain. Previously, an implant filled with autologous bone graft was used to facilitate intervertebral fusion and maintain disc height ${ }^{4,5)}$. However, because harvesting autologous bone grafts causes several complications, various cages, comprised of carbon poly-ether-ether-ketone (PEEK) and titanium alloy, and intervertebral spacer substitutes, such as bioactive glass-ceramics, have been studied ${ }^{6,7)}$, and artificial implantation of the intervertebral disc space has become the more conventional PLIF method $^{4,5}$. Since the first experimental use of titanium or PEEK cages for human lumbar interbody fusion in the $1990 \mathrm{~s}^{8,9)}$, various interbody fusion cages have been used to promote fusion and maintain spinal alignment in lumbar interbody fusion surgeries ${ }^{9-11)}$. This method has many advantages for recovering intervertebral disc height, improving impaired lumbar lordosis, and indirect decompression of the neural structure, and also allows the anterior column to transmit weight effectively ${ }^{4,8)}$. In elderly individuals, failure of instruments, such as cage subsidence and screw loosening, and nonunion because of poor bone quality of autologous bone graft due to osteoporosis are great concerns after PLIF ${ }^{10}$. Biological approaches to enhance bone quality and strength, including pharmacological, cell, and gene therapies, have been used ${ }^{12)}$, and various improvements in implant materials and shapes have also been made ${ }^{10)}$. The mechanical strength of ceramic spacers is twice that of hydroxyapatite ${ }^{13)}$. Previous in vitro and in vivo studies showed that bioactive ceramic spacers possess high bioactivity and chemical bonding ability ${ }^{14)}$ and have found that the fusion rates and clinical results of bioactive ceramic spacers spacers were similar to those of titanium cages, implying their safety ${ }^{15-17}$. The most conventionally used interbody fusion cage is a PEEK cage filled with autogenous bone into the disc space10 because the supporting force of the PEEK cage is more similar to that of cortical bone than a titanium cage ${ }^{18)}$. and the titanium cage can cause subsidence because it has a stress shielding effect and elasticity differences ${ }^{18)}$. Many scientists are working on new material fusion cage spacers, which can replace autologous bone spacers. Ideal interbody fusion cages have satisfactory mechanical hardness, accelerate bony fusion, and are biologically harmless ${ }^{15}$, bone grafts. This autografted bone is typically obtained from the patient's iliac crest or local bone found at the fusion site. However, since the autograft is taken from the patient, it is limited in volume and has the potential for associated donor site morbidity. Owing to these limitations, a variety of alternatives to autogenous grafts have been developed.

For lumbar interbody fusion, intervertebral cages are commonly used with a bone graft, including autologous bone, allogenic bone, or bone graft extenders. Calcium phosphate ceramics, such as hydroxyapatite, beta-tricalcium phosphate, and beta-calcium pyrophosphate, have been considered bone graft extenders that accelerate spinal bony fusion ${ }^{13,17,19)}$. However, the bioactive bone graft expanders are limitated due to their weak mechanical characteristics, including brittleness, poor fatigue resistance, and anisotropy ${ }^{13,19)}$. Using only bioactive materials for lumbar interbody fusion generates severe weaknesses in the load-bearing features of the intervertebral space. To overcome these limitations, intervertebral spacer-coated bioactive ceramics can improve the mechanical strength. These bioactive glass-ceramics build apatite layers with the physiological conditions of the bone. They chemically bond directly to the bone, which can enhance the bone-bonding strength ${ }^{16,19,20)}$. Bioactive glass-ceramics are known to chemically bind to the bone, forming a carbohydroxy apatite layer. The $\mathrm{CaO}-\mathrm{SiO}_{2}-\mathrm{P}_{2} \mathrm{O}_{5}-\mathrm{B}_{2} \mathrm{O}_{3}$ (NovoMax, CGBio Inc., Seoul, Korea) bioactive glass-ceramic spacer improves the osteoblastic differentiation of human mesenchymal stem cells ${ }^{16)}$ and enhances the osseointegration of implants when coating the surface ${ }^{21,22)}$. Previous studies have shown that bioactive glass-ceramics could replace conventional titanium cages. NovoMax cages demonstrated similar lumbar fusion rates and clinical outcomes compared to conventional cages ${ }^{5,15)}$. Furthermore, some articles showed that NovoMax cages could be considered a novel intervertebral spacer to achieve successful spinal fusion without safety concerns for long-term use ${ }^{5,15)}$. As PEEK cages are more physiologically similar to natural bone than titanium cages ${ }^{18)}$, we considered the control group as the one using peek cages. The authors want to compare $\mathrm{CaO}-\mathrm{SiO}_{2}-\mathrm{P}_{2} \mathrm{O}_{5}-\mathrm{B}_{2} \mathrm{O}_{3}$ (NovoMax) with the PEEK cage in terms of clinical efficacy and safety in patients that are widely used for PLIF surgery in the clinical field. For clinical safety and considering the advancement of NovoMax, the authors simulataneously used one bioactive ceramic cage and one conventional PEEK cage at the time of first use. This study compared the results between different cages, even in the same disc space of the spine, and between patients receiving this hybrid model with those receiving only conventional PEEK cages. 


\section{MATERIALS AND METHODS}

\section{Patient Selection}

The institutional review board approved this study, which was conducted at the Armed Forces Capital Hospital (AFCH-19IRB-033). This retrospective analysis included data from patients who underwent PLIF surgery in our hospital from 2015 to 2017. Informed consent was obtained from all patients who underwent PLIF surgery who agreed to use either a hybrid of one PEEK cage and one bioactive cage or PEEK cages prior to PLIF surgery. A total of 188 patients who underwent lumbar fusion surgery were enrolled in this study. The experimental group was defined as the hybrid group (H group), and was composed of patients who received a PEEK cage and a bioactive ceramic interbody spacer. The $\mathrm{H}$ group included 19 patients with 21 lumbar disc levels. The control group was defined as the PEEK group (P group); composed of patients who received only PEEK cages, and included 16 patients with 18 disc levels (Fig. 1). The patients who underwent PLIF surgery in the hybrid group were treated with the bioactive ceramic interbody spacer, NovoMax (CGBio Inc., Seoul, Korea) and LP PEEK cages (Medyssey Inc., Chungcheongbuk-do, Korea). The patients in the $\mathrm{P}$ group were treated with two PEEK cages at the disc level. The indications for fusion surgery were as follows: (1) patients who had severe back pain due to severe degenerative disc change; (2) patients who had intractable radiculopathy due to herniated disc or severe foraminal stenosis; (3) instability caused by spondylolisthesis or traumatic burst fracture; and (4) postlaminectomy syndrome. Exclusion criteria were anterior or lateral lumbar fusion surgery, circumferential fusion surgery, fusion due to malignancy, or a follow-up period of less than three months. Patients with liver, kidney, or metabolic disease were also excluded.

\section{Surgical Procedure}

For the conventional PLIF approach, patients were placed in the supine position, and PLIF was performed using a pedicle screw Iliad screw system (Medyssey Inc., Chungcheongbuk-do, Korea) and implantations. The surgery was performed by a single surgeon ( $\mathrm{SH}$ Yoon) in the AFCH. After the midline incision and periosteal dissection, bilateral hemilaminectomy and removal of the superior auricular process at the target level were performed. Total discectomy was also performed. Only two PEEK cages were used in the $\mathrm{P}$ group. On the other hand, the $\mathrm{H}$ group used one PEEK cage and one NovoMax cage, one on each side of the fusion level. Implantation cage packing with an autologous bone chip and allograft bone was inserted. Pedicle screws and rods were inserted and assembled.

\section{Clinical and Radiologic Outcome Measurement}

At the outpatient clinical follow-up, the visual analogue scale (VAS) pain assessment was evaluated. Patients who underwent PLIF surgery underwent a serial radiologic follow-up at 1, 3, 6, 9, and 12 months with dynamic L-spine X-ray and L-spine CT every three months. The authors observed and analyzed the area of fusion with the vertebral endplate by means of osteolysis and subsidence of the implant cage. After one year of follow-up, radiologic assessments were performed every six months. The patients were followed-up until complete fusion was achieved.

To confirm bone union at the surgical site, the degree of fusion was confirmed on CT based on the fusion grade system proposed by Tan et al. ${ }^{23)}$ As all CT scans were performed in 3D, confirmation of bone bridges in all coronal and sagittal directions was possible ${ }^{24)}$. In this study, lumbar lateral dynamic radiography was performed to determine complete fusion when the Cobb's angle ${ }^{25)}$ at the fusion level was less than $3^{\circ}$ and the change in the distance of the in-

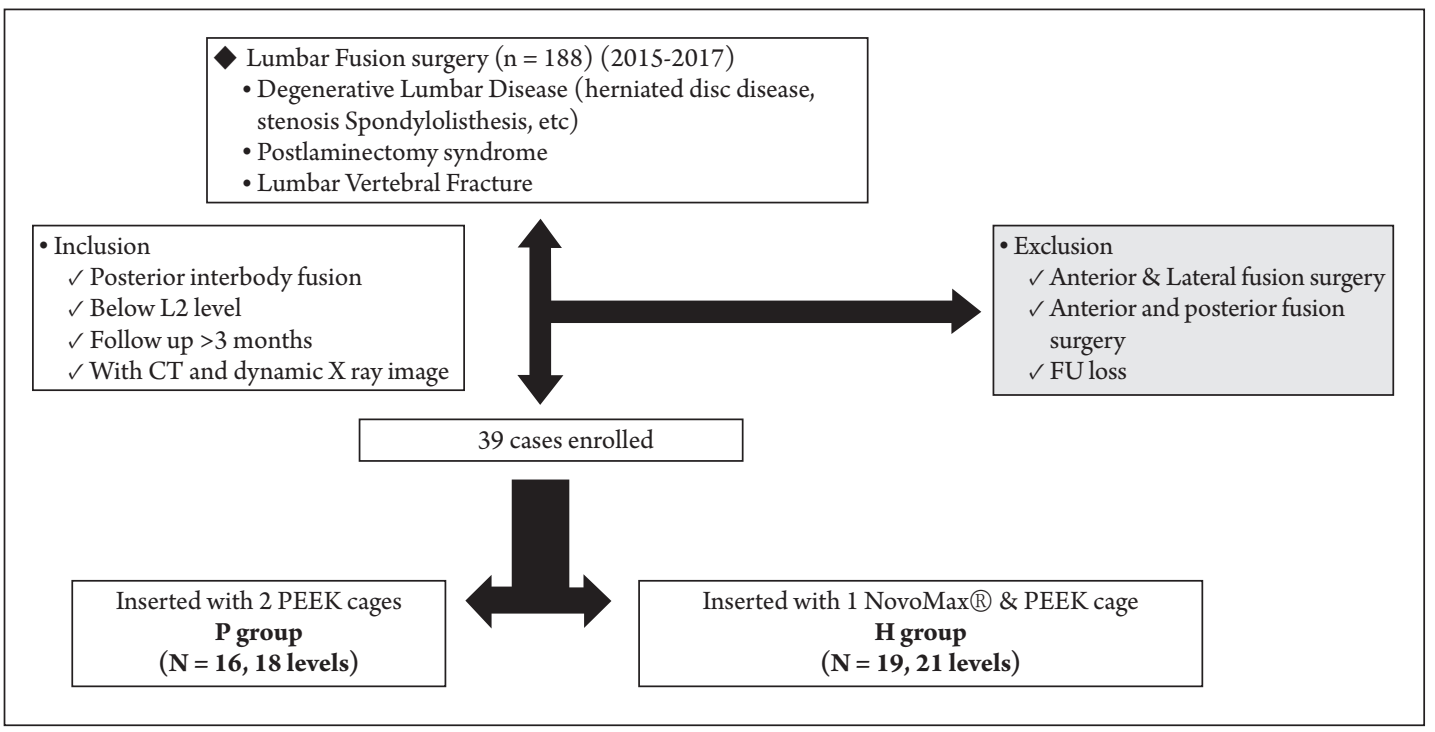

Fig. 1. Study flow diagram. Inclusion and exclusion criteria are shown. FU: Follow-up, PEEK: Poly-ether-ether-ketone, CT: Computed tomography 
terspinous ligament was $<3 \mathrm{~mm}$.

CT images were used to monitor the extent of bone union, osteolysis, osteosclerosis, NovoMax fragmentation, cage slippage, and instrument failure around the union segment (Fig. 2).

\section{Statistical Analysis}

Statistical analysis was performed using IBM SPSS Statistics version 21 (IBM, Armonk, NY, USA). The Kolmogorov-Smirnov test was performed for normality testing. Based on this result, the paired t-test was used for the parameter test to compare the two groups. The Mann-Whitney U test was performed to compare non-parametric continuous variables, and Fisher's exact probability test was used to compare categorical variables between the $\mathrm{P}$ and $\mathrm{H}$ groups. For the evaluation of clinical outcomes, the Wilcoxon signed-rank test was performed to compare the two groups.

Since the fusion was determined through regular observation, Kaplan-Meier survival analysis was performed to analyze the time-series change, and the relative risk of the fusion of risk factors was evaluated using the Cox proportional hazard model. The significance level was set at $\mathrm{p}<0.05$.

\section{Result}

\section{General Aspects}

This retrospective study was conducted from January 1, 2015, to December 31,2017 , on 39 (20.7\%) of the 188 patients who met the inclusion criteria in a single institution (Fig. 1). The patients' demographic and operative data for each group are shown in Table 1. The $\mathrm{H}$ and $\mathrm{P}$ groups demonstrated similar ages, premedical history, and smoking history (Table 1). All patients in this study were male soldiers. There was a statistically significant difference in the mean age between the two groups ( $\mathrm{P}$ group: $45.22 \pm$ 9.15 vs $H$ group: $50.71 \pm 4.78 ; \mathrm{p}=0.032$ ) There was no significant difference in the mean follow-up duration between the $\mathrm{H}$ group $(31.25 \pm 14.84$ months $)$ and the $\mathrm{P}$ group $(24.56 \pm 11.89$ months $)(p=0.118)$. Most patients $(84.6 \%)$ in both groups un- derwent PLIF surgery at the L4-5 and L5-S1 levels. The most common underlying diagnosis for PLIF surgery was postlaminectomy syndrome in 20 cases (51.3\%), followed by spondylolisthesis in 11 cases $(28.2 \%)$, and spinal stenosis in 8 cases.

\section{Clinical and Radiologic Outcome}

\section{Clinical outcome}

Patients pre- and postoperative low back pain level according to the VAS was $5.36 \pm 1.04$ and $1.62 \pm 1.82$, respectively. The preand postoperative VAS level of leg pain were $7.13 \pm 1.10$ and 2.00 \pm 1.67 , respectively. There were no postoperative deaths. One case in the $\mathrm{H}$ group experienced a postoperative infection; the screw was removed after complete fusion, and antibiotics were administered intravenously and orally for 52 weeks. In groups $\mathrm{P}$ and $\mathrm{H}$, worsening of lower extremity pain due to degenerative changes ad-

Table 1. Demographic data

\begin{tabular}{lccc}
\hline Category & P group & H group & p value \\
\hline Age(year) & $45.22 \pm 9.15$ & $50.71 \pm 4.78$ & 0.032 \\
Sex & & & \\
$\quad$ Male & 18 & 21 & \\
Follow up Period(month) & $25.61 \pm 12.30$ & $31.19 \pm 15.39$ & 0.185 \\
Height(cm) & $175.06 \pm 4.57$ & $171.67 \pm 5.01$ & 0.048 \\
Weight(kg) & $82.28 \pm 9.23$ & $78.48 \pm 13.30$ & 0.297 \\
Level of surgery & & & \\
L3/4 & 1 & 5 & \\
L4/5 & 10 & 10 & \\
L5/S1 & 7 & 6 & \\
Smoking & & & \\
Smoker & 12 & 10 & \\
Ex-smoker & 5 & 3 & \\
Current Nonsmoker & 1 & & \\
Comorbidity & 6 & & \\
DM & & & \\
HTN & 5 & & \\
\hline
\end{tabular}

DM: Diabetes mellitus, HTN: Hypertension.
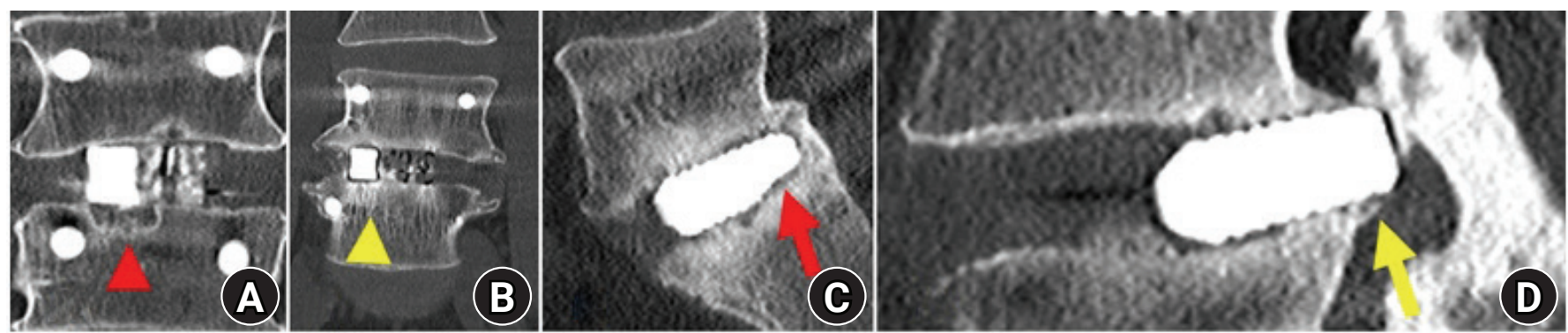

Fig. 2. Computed Tomograph images showing osteolysis (A), osteosclerosis (B), cage fragmentation (C), and slippage (D). 
jacent to the fusion site was observed 2 and 2.5 years after the operation, and symptoms were improved by decompression and fusion surgery through fusion segment extension.

\section{Radiologic outcome}

The degree of fusion of each group was compared and analyzed according to the fusion fycriteria presented in the methodology. According to this analysis, there were 14 cases (72.2\%) in group $\mathrm{P}$ and 15 cases $(71.4 \%)$ in group $\mathrm{H}$ with confirmed complete union during the observation period. There was no statistical difference between the two groups when Fisher's exact probability test was used $(\mathrm{p}=0.62)$. During the observation period, osteolysis showing cystic changes in the endplate was observed in 9 cases $(81.8 \%)$ in the $\mathrm{P}$ group and 21 cases (75\%) in the H group; there was no statistical difference between the two groups when Fisher's exact probability test was used $(\mathrm{p}=0.153)$. Osteosclerosis, a change observed as a high-density change on CT, occurred in most of the subjects $(94.87 \%, 37 / 39)$, and cage subsidence occurred in eight cases in each of the two groups, but there was no difference in incidence between the groups $(\mathrm{p}=0.752)$.

According to the Kaplan-Meier life table, the occurrence of complete fusion was higher in Group $\mathrm{H}$ until approximately 22 months, but was higher in Group P after 22 months (Fig. 3A). Using the occurrence of osteolysis as a covariate in the Cox proportional hazard model, the relative risk of incomplete fusion in both $\mathrm{P}$ and $\mathrm{H}$ groups was increased by 5.41 times $(\mathrm{p}=0.004,95 \% \mathrm{CI}$ 1.704-17.204) (Fig. 3B, C).

\section{Complication related to instrument}

Implant-related complications occurred in both groups. Especially in the $\mathrm{H}$ group, significant failures were observed, such as cage subsidence, fragmentation and slippage. However, implant failure did not cause severe neurological deficits or instability of the fusion site. In addition, there were no revision surgeries related to the implant-related complications.

During the long-term follow-up period, there were four instrument failures related to screw and rod fixation. In the $\mathrm{H}$ group, two patients underwent revision surgery for posterior screw fixation because of screw or rod breakage at the fusion level. In the P group, a patient underwent a second operation due to screw breakage.

\section{Discussion}

This study comparing fusion outcomes between conventional PEEK and hybrid models revealed comparable outcomes. Some studies using bioactive ceramic cages have revealed a more complete fusion outcome compared with that found in this study, ${ }^{5,15}$.
This difference in fusion outcome could be due to various reasons related to the PLIF surgery. The patients in this study used a bioac-
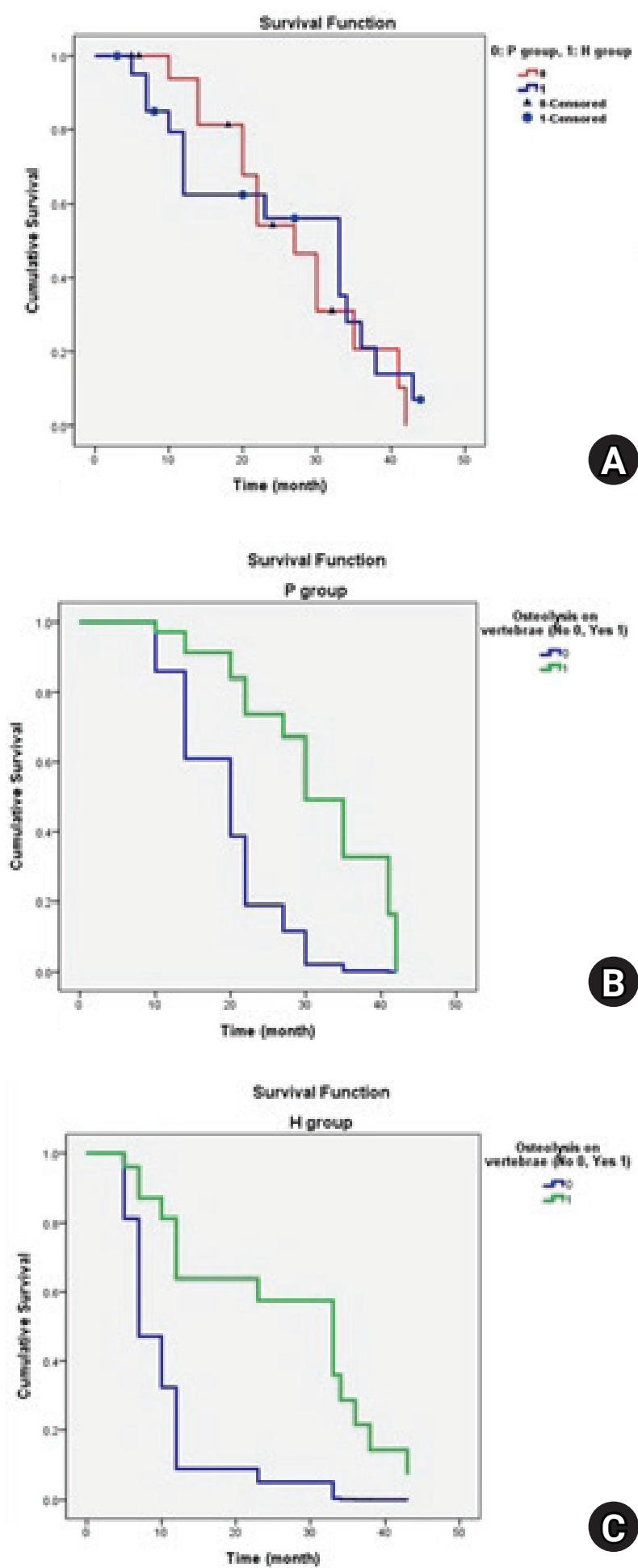

Fig. 3. Kaplan-Meier life table (A) showing complete fusion as survival rate through time course between Groups $\mathrm{P}$ and $\mathrm{H}$. In the Cox proportional hazard model, complete fusion rate concerning occurrence of osteolysis between $\mathrm{P}$ group (B) and $\mathrm{H}$ group (C). 
tive ceramic cage on one side of the fusion level and a conventional PEEK cage on the other. This strategy may have a less potent effect on fusion. However, there is no strong evidence that hybrid cage usage is inferior to conventional ages because osteolytic or osteosclerosis lesions were not found in the NovoMax cage side. As mentioned in previous studies ${ }^{5,15)}$, if the bioactive cage accelerates bone fusion, the affected endplate margin near the bioactive ceramic cage side could have more bony signal changes. However, there was no significant difference in the endplate change.

Previous studies have shown that bioactive materials can accelerate bony fusion in nearby cages; however, there was no significant complete fusion rate and period difference during the follow-up ${ }^{20,23)}$. In our study, bioactive ceramic cages had more implant complications, such as spacer migration, fragmentation, and subsidence. Although this complication did not change the patient's hospital course, it could lead to critical complications, including fusion failure and compression of the thecal sac. Therefore, if a bioactive ceramic cage is used, careful serial image follow-up should be performed.

There are limitations inherent in this study. The total number of patients was smaller than that in the previous studies, and the follow-up period was too short to confirm fusion in some of the patients. To overcome this pitfall, we analyzed this result with the Kaplan-Meier life table and Cox proportional hazard model to show the relative risk and probability of fusion over time.

When NovoMax was used, bony fusion tended to progress more rapidly the first 22 months after surgery than with conventional PEEK cages without a statistical difference; otherwise, after 22 months, the fusion was delayed. It is feared that the possibility of non-union is somewhat higher after after 22 months. When accompanied by osteolysis, the probability of nonunion increased by more than 5.4 times in the Cox proportional hazard model. In this study, NovoMax and PEEK cages were used together at the same level, which was not very helpful when comparing superiority.

Previous literature ${ }^{5,15)}$ related to NovoMax did not mention the occurrence of osteolysis, osteosclerosis, cage slippage, and fragmentation. There is a lack of evidence on what to pay attention to. Through this study, some of the limitations and complications of this method were observed, which could enhance the surgical decision process and perioperative care.

It should be noted that owing to NovoMax's material and morphological properties, (ie., smooth and low-resistance surface) patients may experience breakage and pull out during the operative and follow-up periods.

\section{Conclusion}

In PLIF surgery, a bioactive glass spacer showed a fusion rate similar to that of PEEK cages. Using NovoMax could result in osteolysis, cage migration, fragmentation, or subsidence. However, it is worth remembering that these abnormal findings were not clearly reported in previous clinical trials. The use of NovoMax is expected to be an alternative to conventional interbody fusion with PEEK cages, but close attention should be paid to the problems that may accompany it as retardation of accomplishing complete fusion goes on.

\section{NOTES}

\section{Conflict of interest}

No potential conflict of interest relevant to this article was reported.

\section{Ethics committee approval}

This study was approved by the IRB at Armed Forces Capital Hospital. IRB number for this study is:. AFCH-19-IRB-033. All ethical guideline rules were followed to protect patient privacy.

\section{Funding statement}

This research received no specific grant from any funding agency in the public or commercial or sectors.

\section{REFERENCES}

1. Kanayama M, Cunningham BW, Haggerty CJ, Abumi K, Kaneda K, McAfee PC. In vitro biomechanical investigation of the stability and stress-shielding effect of lumbar interbody fusion devices. J Neurosurg 2000;93(2 Suppl):259-265.

2. Kumar N, Judith MR, Kumar A, Mishra V, Robert MC. Analysis of stress distribution in lumbar interbody fusion. Spine 2005;30:1731-1735.

3. Polly DW, Klemme WR, Cunningham BW, Burnette JB, Haggerty CJ, Oda I. The biomechanical significance of anterior column support in a simulated single-level spinal fusion. J Spinal Disord 2000; 13:58-62.

4. Blumenthal SL, Ohnmeiss DD. Intervertebral cages for degenerative spinal diseases. Spine J 2003;3:301-9.

5. Lee JH, Kim SK, Kang SS, Han SJ, Lee C-K, Chang BS. A longterm follow-up, multicenter, comparative study of the radiologic, and clinical results between a cao-sio2-p2o5-b2o3 bioactive glass ceramics (bgs-7) intervertebral spacer and titanium cage in 1-level posterior lumbar interbody fusion. Clin Spine Surg 
2020;33:E322-E329.

6. Abdul QR, Qayum MS, Saradhi MV, Panigrahi MK, Sreedhar V. Clinico-radiological profile of indirect neural decompression using cage or auto graft as interbody construct in posterior lumbar interbody fusion in spondylolisthesis: Which is better? J Craniovertebr Junction Spine 2011;2:12-16.

7. Brislin B, Vaccaro AR. Advances in posterior lumbar interbody fusion. Orthop Clin North Am 2002;33:367-374.

8. Makino T, Takenaka S, Sakai Y, Yoshikawa H, Kaito T. Comparison of Short-Term Radiographical and Clinical Outcomes After Posterior Lumbar Interbody Fusion With a 3D Porous Titanium Alloy Cage and a Titanium-Coated PEEK Cage. Global Spine J 2020;2192568220972334.

9. de Kunder SL, Rijkers K, Caelers I, de Bie RA, Koehler PJ, van Santbrink H. Lumbar interbody fusion: a historical overview and a future perspective. Spine (Phila Pa 1976) 2018;43:11611168.

10. Vadapalli S, Sairyo K, Goel VK, Robon M, Biyani A, Khandha $\mathrm{A}$, et al. Biomechanical rationale for using polyetheretherketone (PEEK) spacers for lumbar interbody fusion-A finite element study. Spine (Phila Pa 1976) 2006;31:E992-E998.

11. Kanayama M, Cunningham BW, Haggerty CJ, Abumi K, Kaneda K, McAfee PC. In vitro biomechanical investigation of the stability and stress-shielding effect of lumbar interbody fusion devices. J Neurosurg 2000;93(2 Suppl):259-265.

12. Tan GH, Goss BG, Thorpe PJ, Williams RP. CT-based classification of long spinal allograft fusion. Eur Spine J 2007;16: 1875-1881.

13. Kokubo T, Ito S, Huang Z, Hayashi T, Sakka S, Kitsugi T, et al. $\mathrm{Ca}, \mathrm{P}$-rich layer formed on high-strength bioactive glass-ceramic A-W.J Biomed Mater Res 1990;24:331-343.

14. Lee JH, Nam H, Ryu HS, Seo JH, Chang BS, Lee CK. Bioactive ceramic coating of cancellous screws improves the osseointegration in the cancellous bone. J Orthop Sci 2011;16:291-7.

15. Lee JH, Kong CB, Yang JJ, Shim HJ, Koo KH, Kim J, et al. Comparison of fusion rate and clinical results between $\mathrm{CaO}-\mathrm{SiO} 2-$ P2O5-B2O3 bioactive glass ceramics spacer with titanium cages in posterior lumbar interbody fusion. Spine J 2016;16:1367-
1376.

16. Lee JH, Seo JH, Lee KM, Ryu HS, Baek HR. Fabrication and evaluation of osteoblastic differentiation of human mesenchymal stem cells on novel CaO-SiO2-P2O5-B2O3 glass-ceramics. Artificial organs 2013;37:637-647.

17. Ohtsuki C, Kamitakahara M, Miyazaki T. Bioactive ceramic-based materials with designed reactivity for bone tissue regeneration. J R Soc Interface 2009;6(suppl3):S349-S360.

18. Chou YC, Chen DC, Hsieh WA, Chen WF, Yen PS, Harnod T, et al. Efficacy of anterior cervical fusion: comparison of titanium cages, polyetheretherketone (PEEK) cages and autogenous bone grafts. J Clin Neurosci 2008;15:1240-5.

19. Lee JH, Jeung UO, Jeon DH, Chang BS, Lee CK. Quantitative comparison of novel cao-sio2-p2o5-b2o3 glass-ceramics (bgs7) with hydroxyapatite as bone graft extender in rabbit ilium. Tissue Eng Regen Med 2010;7:540.

20. Lee JH, Nam H, Ryu HS, Seo JH, Chang BS, Lee CK. Bioactive ceramic coating of cancellous screws improves the osseointegration in the cancellous bone. J Orthop Sci 2011;16(3):291-297.

21. Lee JH, Ryu HS, Seo JH, Chang BS, Lee CK. A 90-day intravenous administration toxicity study of $\mathrm{CaO}-\mathrm{SiO} 2-\mathrm{P} 2 \mathrm{O} 5-\mathrm{B} 2 \mathrm{O} 3$ glass-ceramics (BGS-7) in rat. Drug Chem Toxicol 2010;33: $38-47$.

22. Koo KH, Hwang C, Lee JH, Chang BS, Lee CK. Treatment of bone defects in rabbit tibiae using $\mathrm{CaO}-\mathrm{SiO} 2-\mathrm{P} 2 \mathrm{O} 5-\mathrm{B} 2 \mathrm{O} 3$ bioactive ceramics: radiological, biomechanical, and histological evaluation. Tissue Eng Regen Med 2009;6:811.

23. Tan G, Goss B, Thorpe P, Williams R. CT-based classification of long spinal allograft fusion. Eur Spine J 2007;16:1875-1881.

24. Lee JH, Jeon DW, Lee SJ, Chang BS, Lee CK. Fusion rates and subsidence of morselized local bone grafted in titanium cages in posterior lumbar interbody fusion using quantitative three-dimensional computed tomography scans. Spine 2010;35:14601465.

25. Lee HS, Lee JH, Lee JH. A comparison of dynamic views using plain radiographs and thin-section three-dimensional computed tomography in the evaluation of fusion after posterior lumbar interbody fusion surgery. Spine J 2013;13:1200-1207. 